\title{
Pharmacokinetics of liver-targeted docetaxel liposomes modified with 6-O-acyl-D-galactose esters in rabbits
}

\author{
WEI WU ${ }^{1,2}$, YI CHENG ${ }^{1}$, BO-HONG GUO ${ }^{1}$ and QIONG WU ${ }^{1}$ \\ ${ }^{1}$ School of Chinese Materia Medica, Guangzhou University of Traditional Chinese Medicine, Guangzhou, Guangdong 510006; \\ ${ }^{2}$ School of Pharmacy, Guilin Medical University, Guilin, Guangxi 541004, P.R. China
}

Received March 19, 2014; Accepted May 12, 2014

DOI: $10.3892 /$ br.2014.285

\begin{abstract}
The purpose of the present study was to investigate the pharmacokinetics of docetaxel liposomes modified with 6-O-acyl-D-galactose esters (Gal-DOC-L) in rabbits. A simple, rapid and sensitive high-performance liquid chromatography (HPLC) method was developed for the determination of docetaxel. Gal-DOC-L was intravenously administered to rabbits with norethisterone as the internal standard and the blood samples were collected from ear marginal veins at $0.083,0.25,0.5,1,2,4,6,8,12,16$ and $24 \mathrm{~h}$ after treatment. The plasma concentration of docetaxel was determined by HPLC and the pharmacokinetic parameters were calculated. Docetaxel injection (DOC-I) was studied simultaneously. The results showed that the area under the curve ${ }_{(0-\infty)}, t_{1 / 2 \alpha}$ and $t_{1 / 2 \beta}$ of Gal-DOC-L was significantly higher, while the total body clearance was lower than that of DOC-I. The results indicated that Gal-DOC-L was able to maintain a relatively high blood concentration in vivo and prolong the treatment time.
\end{abstract}

\section{Introduction}

Liposomes are commonly used as pharmaceutical delivery vehicles to improve therapeutic efficacy and reduce drug toxicity (1). Encapsulation of drugs into liposomes can prolong the time course of the drug effect and improve the stability of drugs in vitro and in vivo (2). However, rapid uptake of liposomes in vivo by cells of the mononuclear phagocytic system (MPS) has restricted their therapeutic utility (3). Surface modification of liposomes by carbohydrates, glycolipids or polymers to form long-circulating liposomes can alter their pharmacokinetic pattern and reduce MPS uptake (4). Furthermore, liposomes that have been modified with glycolipid-targeted ligands

Correspondence to: Professor Yi Cheng, School of Chinese Materia Medica, Guangzhou University of Traditional Chinese Medicine, 232 East of Waihuan Road, University Town, Guangzhou, Guangdong 510006, P.R. China

E-mail: yicheng81@126.com

Key words: pharmacokinetics, docetaxel liposomes, 6- $O$-acyl-D-galactose, high-performance liquid chromatography are promising as a long-circulating and tumor-targeting carrier system (5). Asialoglycoprotein receptor (ASGPR) is a high-capacity galactose-binding receptor expressed on the surface of hepatocytes (6) that possesses characteristics that can specifically recognize glycoproteins that possess exposed terminal galactose or lactose residues (7). Liposomes modified with galactose derivatives can be recognized and bound by ASGPR in liver parenchyma cells, and significantly improve the liver-targeted effect of liposome drugs (8). This is crucial in the development of novel drugs in the treatment of hepatic carcinoma (9). Therefore, liposomes modified with galactose esters not only avoid the uptake of MPS, but also improve the targeting effect of liposomal carriers.

In our previous study (10), 6-O-acyl-D-galactose esters were synthesized by the enzyme-catalyzed esterification of D-galactose and vinyl stearate (Fig. 1).

The anti-cancer drug docetaxel was used as a model drug, and docetaxel liposomes modified with 6- $O$-acyl-D-galactose esters (Gal-DOC-L) were successfully prepared as a novel formulation for liver cancer treatment (11). In the present study, the pharmacokinetic characteristics of docetaxel following intravenous administration of Gal-DOC-L in rabbits was investigated, and the results were compared with docetaxel injection (DOC-I).

\section{Materials and methods}

Materials. Docetaxel (purity, 99\%) was purchased from the Beijing Norzer Pharmaceutical Co., Ltd. (Beijing, China). Norethisterone was purchased from the National Institute for the Control of Pharmaceutical and Biological Products (Beijing, China). DOC-I was purchased from the Jiangsu Hengrui Medicine Co., Ltd. (Jiangsu, China). Gal-DOC-L were prepared in our laboratory (Department of Pharmaceutics, School of Chinese Materia Medica, Guangzhou University of Traditional Chinese Medicine, Guangzhou, Guangdong, China). Methanol and acetonitrile from the Shandong Yuwang Industrial Co., Ltd. (Shandong, China) were of chromatographic grade. All other reagents were of analytical grade and used without further purification.

Instruments. The Dionex high-performance liquid chromatography (HPLC) system consisted of a PDA-100 detector, a P680 pump and an ASI-100 autosampler (Dionex, Sunnyvale, 
CA, USA). The chromeleon chromatography workstation software was used for data acquisition and processing.

Chromatographic conditions. Chromatographic separation was carried out on a Diamonsil C18 column (250x4.6 mm, $5 \mu \mathrm{m}$; Dikma Technologies, Inc., Radnor, PA, USA) with a EasyGuard C18 Security guard column $(8 \times 4.0 \mathrm{~mm}$ internal diameter; Dikma Technologies, Inc.) maintained at $30^{\circ} \mathrm{C}$. The mobile phase consisted of acetonitrile:water $(55: 45, \mathrm{v} / \mathrm{v})$, at a flow rate of $1.0 \mathrm{ml} / \mathrm{min}$, and the wavelength was set at $230 \mathrm{~nm}$.

Experimental animals. New Zealand rabbits were purchased from the Laboratory Animal Center of Guangzhou University of Chinese Medicine (Guangzhou, Guangdong, China). The rabbits were acclimatized to the laboratory conditions one week prior to the experiment. The rabbits were deprived of food but provided with water ad libitum for $12 \mathrm{~h}$ before the experiment. All experiments that included blood collection from the rabbit ear marginal veins were approved by Guilin Medical University Animal Ethics Committee (Guilin, China).

Sample preparation. In the present study, methyl tertiary-butyl ether was used as a solvent for the extraction of drugs from plasma $(12,13)$. The blood samples $(0.9 \mathrm{ml})$ were collected from the rabbit ear marginal veins at a dose of $10.0 \mathrm{mg}$ docetaxel eq/ $\mathrm{kg}$. Plasma was obtained by centrifugation of the blood at $1,000 \mathrm{x} \mathrm{g}$ for $10 \mathrm{~min}$. Norethisterone $(2.0 \mathrm{mg} / \mathrm{ml}, 100 \mu \mathrm{l})$ as an internal standard was added into $900 \mu \mathrm{l}$ of plasma, and vortexed with $3.0 \mathrm{ml}$ tert-butyl methyl ether for $10 \mathrm{~min}$. Following centrifugation of the mixture at $1,000 \times \mathrm{g}$ for $5 \mathrm{~min}$, the upper organic layer was collected and evaporated to dryness with $\mathrm{N}_{2}$ at $30^{\circ} \mathrm{C}$. The residue was reconstituted in $10 \mathrm{ml}$ of HPLC mobile phase, vortexed for $1 \mathrm{~min}$ and centrifuged at 11,200 x $\mathrm{g}$ for $5 \mathrm{~min}$. The plasma concentration of docetaxel was determined by HPLC.

HPLC method validation. The method of HPLC was validated according to the currently accepted USA Food and Drug Administration (FDA) bioanalytical method validation guidance (14). The following validation characteristics were determined: Specificity, linearity, precision and accuracy.

The specificity was evaluated by comparing retention times obtained in the standard solutions of docetaxel using various samples (blank plasma sample, blank plasma sample spiked with docetaxel and plasma sample following administration of Gal-DOC-L). The calibration curves were constructed with seven concentrations (simultaneously prepared) ranging from 0.1 to $10 \mathrm{mg} / \mathrm{ml}$ for standard solutions of docetaxel. Each concentration level was prepared in triplicate and analyzed three times. Calibration curves were constructed by plotting the concentration of compounds vs. the peak area response. The linearity was evaluated by the least square regression method. The precision was determined by repeatability (intra-day) and intermediate precision (inter-day) and was expressed as the relative standard deviation (\%). The accuracy was tested by replicate analysis of different samples at known concentrations and then compared with the true concentration of a standard, and accuracy was assessed by the recovery percentage.
Table I. Intra- and inter-day precision and accuracy for determination of docetaxel $(n=5)$.

\begin{tabular}{|c|c|c|c|c|}
\hline \multirow{2}{*}{$\begin{array}{l}\text { Concentration, } \\
\mathrm{mg} / \mathrm{ml}\end{array}$} & \multirow{2}{*}{$\begin{array}{l}\text { Added, } \\
\mu \mathrm{g} / \mathrm{ml}\end{array}$} & \multicolumn{2}{|c|}{ RSD, \% } & \multirow[b]{2}{*}{ Recovery (\%) } \\
\hline & & Intra-day & Inter-day & \\
\hline 0.1 & 0.1 & 4.56 & 7.13 & $82.45 \pm 3.01$ \\
\hline 1.0 & 1.0 & 2.17 & 6.39 & $93.22 \pm 0.95$ \\
\hline 10.0 & 10.0 & 3.88 & 4.24 & $86.97 \pm 1.64$ \\
\hline
\end{tabular}

RSD, relative standard deviation.

Table II. Pharmacokinetic parameters of docetaxel following a single intravenous injection of Gal-DOC-L and DOC-I in rabbits with a dose of $10.0 \mathrm{mg} / \mathrm{kg}(\mathrm{n}=6)$.

\begin{tabular}{llrr}
\hline Parameters & Units & \multicolumn{1}{c}{ DOC-I } & Gal-DOC-L \\
\hline $\mathrm{t}_{1 / 2 \alpha}$ & $\mathrm{h}$ & $0.039 \pm 0.005$ & $0.134 \pm 0.017$ \\
$\mathrm{t}_{1 / 2 \beta}$ & $\mathrm{h}$ & $3.848 \pm 0.751$ & $8.891 \pm 1.280$ \\
$\mathrm{~V}_{1}$ & $\mathrm{l} / \mathrm{kg}$ & $0.847 \pm 0.099$ & $2.575 \pm 0.304$ \\
$\mathrm{CL}$ & $\mathrm{l} / \mathrm{kg} \cdot \mathrm{h}^{-1}$ & $3.001 \pm 0.346$ & $0.745 \pm 0.101$ \\
$\mathrm{AUC}$ & $\mathrm{mg} \cdot \mathrm{h} / \mathrm{l}$ & $2.747 \pm 0.357$ & $11.126 \pm 1.643$ \\
$\mathrm{AUC}_{(0-\infty)}$ & $\mathrm{mg} \cdot \mathrm{h} / \mathrm{l}$ & $3.332 \pm 0.402$ & $13.417 \pm 1.468$ \\
$\mathrm{~K}_{10}$ & $\mathrm{~h}^{-1}$ & $3.544 \pm 0.485$ & $0.289 \pm 0.036$ \\
$\mathrm{~K}_{12}$ & $\mathrm{~h}^{-1}$ & $13.591 \pm 1.590$ & $3.644 \pm 0.397$ \\
$\mathrm{~K}_{21}$ & $\mathrm{~h}^{-1}$ & $0.839 \pm 0.123$ & $1.329 \pm 0.178$ \\
\hline
\end{tabular}

Gal-DOC-L, docetaxel liposomes modified with 6- $O$-acyl-D-galactose esters; DOC-I, docetaxel injection.

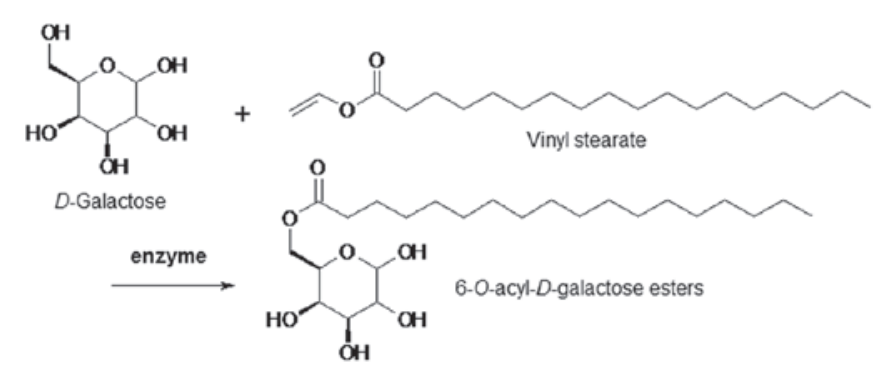

Figure 1. Chemical structures of the enzymatic synthesis.

Pharmacokinetics investigation. Gal-DOC-L or DOC-I were intravenously administered into the ear marginal veins of rabbits at a dose of $10.0 \mathrm{mg}$ docetaxel eq $/ \mathrm{kg}$. The blood samples $(0.9 \mathrm{ml})$ were collected from the ear marginal veins with a heparinized syringe at $0.083,0.25,0.5,1,2,4,6,8$, 12,16 and $24 \mathrm{~h}$ after administration. Plasma was obtained by centrifugation of the blood at $1,000 \mathrm{x} \mathrm{g}$ for $10 \mathrm{~min}$. Norethisterone $(2.0 \mathrm{mg} / \mathrm{ml}, 100 \mu \mathrm{l})$ was added into $900 \mu \mathrm{l}$ of plasma, and vortexed with $3.0 \mathrm{ml}$ tert-butyl methyl ether for $10 \mathrm{~min}$. Following centrifugation of the mixture at $1,000 \mathrm{x} \mathrm{g}$ for $5 \mathrm{~min}$, the upper organic layer was collected and evaporated to dryness with $\mathrm{N}_{2}$ at $30^{\circ} \mathrm{C}$. The residue was 
A

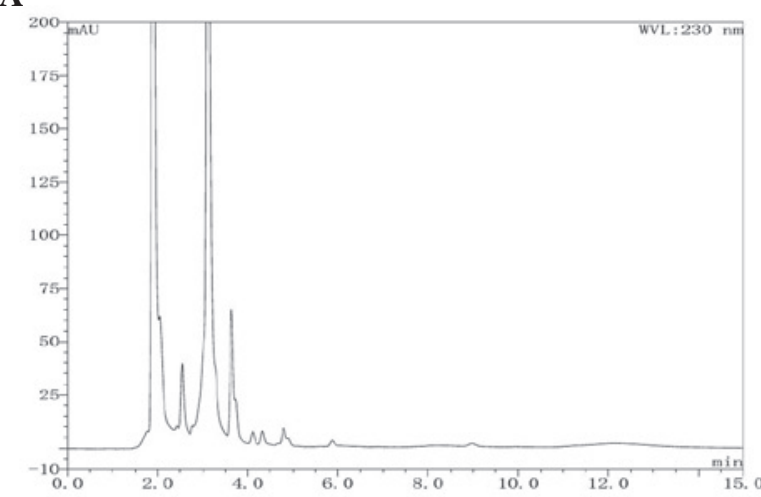

C

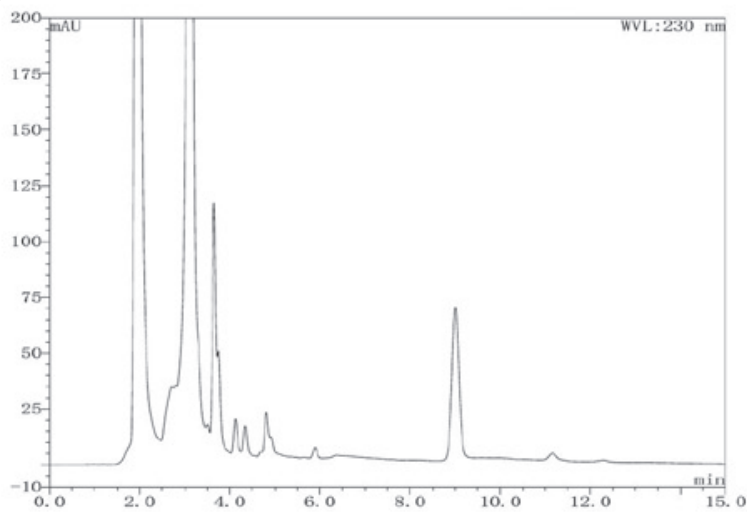

B

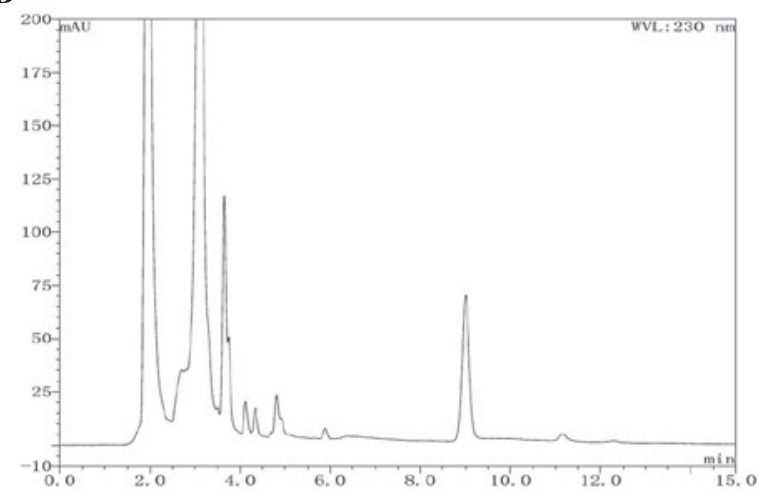

D

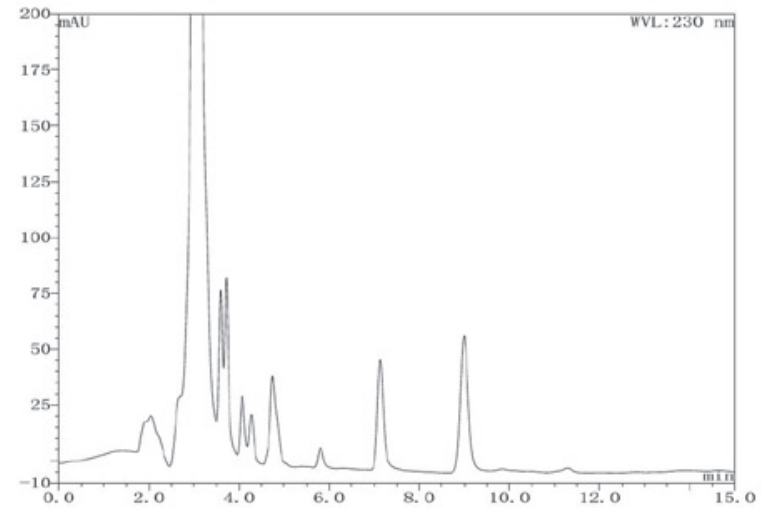

Figure 2. HPLC chromatograms of plasma sample. Chromatograms following the injection of (A) docetaxel, (B) blank plasma sample, (C) blank plasma sample spiked with docetaxel, and (D) plasma sample following administration of Gal-DOC-L. HPLC, high-performance liquid chromatography; Gal-DOC-L, docetaxel liposomes modified with 6-O-acyl-D-galactose esters.

reconstituted in $10 \mathrm{ml}$ of HPLC mobile phase, vortexed for $1 \mathrm{~min}$ and centrifuged at 11,200 x g for $5 \mathrm{~min}$. The plasma concentration of docetaxel was determined by HPLC and pharmacokinetic parameters were calculated with DAS 2.0 software (Mathematical Pharmacology Professional Committee of China, Shanghai, China).

\section{Results and Discussion}

Method validation. The specificity analysis revealed that the developed HPLC method was not affected by interference at the retention time of the analytes. The typical chromatograms of the docetaxel, blank plasma, blank plasma spiked with docetaxel and plasma samples following administration of Gal-DOC-L are shown in Fig. 2.

There was a good linearity in the tested range. The area response was $\mathrm{y}=0.2728 \mathrm{x}-0.0066$ with a correlation coefficient of 0.9992 and confidence intervals at $\mathrm{P}=0.05$. The precision obtained for the repeatability studies and for intermediate precision (expressed as RSD) was in the range between 2 and $8 \%$, which comply with the acceptance criteria proposed (not more $15 \%$ ). The accuracy was expressed as percent recoveries obtained for different docetaxel concentrations. The results indicated that the analytical method has acceptable precision and accuracy (Table I).

Pharmacokinetics investigation. The change in concentration over the time period for docetaxel in plasma following

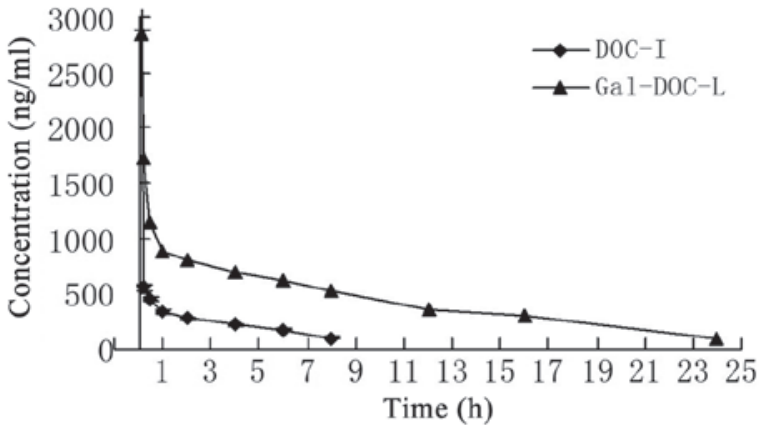

Figure 3. Concentration of docetaxel in plasma at various times following a single intravenous injection in rabbits with a dose of $10.0 \mathrm{mg} / \mathrm{kg}(\mathrm{n}=6)$.

intravenous injection of Gal-DOC-L and DOC-I in rabbits is shown in Fig. 3.

A peak plasma concentration was achieved rapidly following intravenous injection of the docetaxel formulation, with Gal-DOC-L having a drug concentration-time curve similar to DOC-I. The results indicated that docetaxel was rapidly removed from the circulation. By contrast, Gal-DOC-L showed a markedly delayed clearance from the plasma. DOC-I was rapidly removed from the circulating system and was not detected in the plasma $8 \mathrm{~h}$ after intravenous injection. The concentration-time curves for Gal-DOC-L and DOC-I in rabbits were fitted by the two-compartment model, and their pharmacokinetic parameters are shown in Table II. The 
results showed that Gal-DOC-L prolonged $t_{1 / 2 \beta}$ of docetaxel by 2.31-fold in plasma, and improved the area under the curve ${ }_{(0-\infty)}$ values of docetaxel by 4.03 -fold compared to DOC-I. The docetaxel in Gal-DOC-L was eliminated gradually.

In the present study, the prolonged circulation time of Gal-DOC-L in the plasma is possibly due to a reduced interaction with plasma and cell-surface proteins. A possible explanation for the reduced interaction is the steric hindrance effect, which is generated by the surface-grafted 6-O-acyl-D-galactose esters molecules (15). As galactose esters exist on the surface of liposomes, a longer persistence of the liposomes in the blood enabled an improved targeting effect, which enhanced the active targeting function of the liposome. In order to further evaluate the liver targeting effect of Gal-DOC-L, analysis of the tissue distribution in mice is required.

\section{Acknowledgements}

The present study was supported by the Natural Science Foundation of China (NSFC; grant no. 30772790). The authors gratefully acknowledge the financial support by the NSFC.

\section{References}

1. Samad A, Sultana Y and Aqil M: Liposomal drug delivery systems: an update review. Curr Drug Deliv 4: 297-305, 2007.

2. Gabizon A, Shmeeda H and Barenholz Y: Pharmacokinetics of pegylated liposomal Doxorubicin: review of animal and human studies. Clin Pharmacokinet 42: 419-436, 2003.

3. Woodle MC and Lasic DD: Sterically stabilized liposomes. Biochim Biophys Acta 1113: 171-199, 1992.

4. Allen TM, Hansen CB and de Menezes DEL: Pharmacokinetics of long-circulating liposomes. Adv Drug Deliv Rev 16: 267-284, 1995.
5. Cai L, Wang X, Wang W, et al: Peptide ligand and PEG-mediated long-circulating liposome targeted to FGFR overexpressing tumor in vivo. Int J Nanomedicine 7: 4499-4510, 2012.

6. Mamidyala SK, Dutta S, Chrunyk BA, et al: Glycomimetic ligands for the human asialoglycoprotein receptor. J Am Chem Soc 134: 1978-1981, 2012.

7. Ye Z, Houssein HS and Mahato RI: Bioconjugation of oligonucleotides for treating liver fibrosis. Oligonucleotides 17: 349-404, 2007.

8. Li Y, Huang G, Diakur J and Wiebe LI: Targeted delivery of macromolecular drugs: asialoglycoprotein receptor (ASGPR) expression by selected hepatoma cell lines used in antiviral drug development. Curr Drug Deliv 5: 299-302, 2008.

9. Zhou X, Zhang M, Yung B, Li H, Zhou C, Lee LJ and Lee RJ: Lactosylated liposomes for targeted delivery of doxorubicin to hepatocellular carcinoma. Int J Nanomedicine 7: 5465-5474, 2012.

10. Cheng Y, Wu W, Zhang D and Mai YZ: Enzyme-catalyzed synthesis of ASGPR ligand-targeted modifier in non-aqueous medium. Acta Pharmaceutica Sinica 45: 1134-1138, 2010 (In Chinese).

11. Guo BH, Cheng Y, Lin LP, Lin DH and Wu W: Preparation and characterization of galactose-modified liposomes by a nonaqueous enzymatic reaction. J Liposome Res 21: 255-260, 2011.

12. Kuyukina MS, Ivshina IB, Philp JC, Christofi N, Dunbar SA and Ritchkova MI: Recovery of Rhodococcus biosurfactants using methyl tertiary-butyl ether extraction. J Microbiol Methods 46: 149-156, 2001.

13. Matyash V, Liebisch G, Kurzchalia TV, Shevchenko A and Schwudke D: Lipid extraction by methyl-tert-butyl ether for high-throughput lipidomics. J Lipid Res 49: 1137-1146, 2008.

14. FDA C: Guidance for industry: bioanalytical method validation. US Department of Health and Human Services. Food and Drug Administration, Center for Drug Evaluation and Research (CDER), Center for Veterinary Medicine (CV), 2001.

15. Immordino ML, Dosio F and Cattel L: Stealth liposomes: review of the basic science, rationale, and clinical applications, existing and potential. Int J Nanomedicine 1: 297-315, 2006. 